\title{
Size-Dependent Coherent-Phonon Plasmon Modulation and Deformation Characterization in Gold Bipyramids and Nanojavelins
}

\author{
Matthew S. Kirschner, ${ }^{1,2}$ Clotilde M. Lethiec, ${ }^{1}$ Xiao-Min Lin, ${ }^{2}$ George C. Schatz, ${ }^{1}$ Lin X. \\ Chen, ${ }^{1,3}$ and Richard D. Schaller ${ }^{1,2}$ \\ 1. Department of Chemistry, Northwestern University, 2145 Sheridan Rd., Evanston, IL 60208 \\ 2. Center for Nanoscale Materials, Argonne National Laboratory, 9700 Cass Ave., Argonne, IL 60439 \\ 3. Chemical Sciences and Engineering Division, Argonne National Laboratory, 9700 Cass Ave., Argonne, IL 60439
}

\section{Supporting Information}

\section{Transient Absorption Data Fitting}

For gold bipyramids and nanojavelins, the longitudinal plasmon resonance is well modeled using Lorentzian functions. Since the photoexcited particles also present fairly low size- and aspect-ratio dispersity, the transient optical signal can be approximated as two Lorentzian functions $\mathrm{S1-S4}$

$$
\Delta A(\omega, t)=a(t)\left(\left(\frac{\Gamma_{0}}{\left(\omega-\omega_{0}\right)^{2}+\left(\frac{\Gamma_{0}}{2}\right)^{2}}\right)-\frac{\Gamma_{\varepsilon}(t)}{\left.\left(\omega-\omega_{\varepsilon}(t)\right)^{2}+\left(\frac{\Gamma_{\varepsilon}(t)}{2}\right)\right)^{2}}\right)
$$

where $t$ is time, $\omega$ is frequency, $\Gamma_{0}$ and $\omega_{0}$ are the static extinction width and center respectively taken from static spectra, and $\mathrm{a}(\mathrm{t}), \Gamma_{\varepsilon}(t)$, and $\omega_{\varepsilon}(t)$ are constants that are allowed to vary in time which represent a normalization factor, the resonance linewidth, and center frequency.

\section{Phonon Dynamics Interpretation}

As discussed in the main text, the time evolution of the LSPR center wavelength was modeled using the equation:

$$
\omega_{\varepsilon}(t)=\sum_{i} \beta_{i} e^{-t / \tau_{1 i}} e^{-\left(\frac{t}{\tau_{2 i}}\right)^{2}} \sin \left(\frac{2 \pi t}{T_{i}}+\varphi_{i}\right)+\omega_{0}+\alpha e^{-t / \tau_{c o o l}}
$$

which includes for a given phonon mode $i$, the maximum coherent phonon displacement $\beta$, homogeneous lifetime $\tau_{1}$, inhomogeneous lifetime due to nanoparticle polydispersity $\tau_{2}$, the period of the acoustic vibrations $T$, and phase shift $\varphi$, along with the static LSPR position $\omega_{0}$, a lattice heating term $\alpha$, and a lattice cooling lifetime $\tau_{\text {cool }}$. While fitting the data, $T, \varphi, \omega_{0}$, and $\tau_{2}$ were kept constant across excitation power. At first, we allowed these parameters to vary, but they exhibited no apparent power dependent trends and so they were kept constant to increase the stability of fitting the remaining parameters. The one exception was sample B, where $\omega_{0}$, was allowed to vary in order to improve the quality of the fit. This variance was likely caused by sample inhomogeneity 
and was still quite small changing only by a few wavenumbers. The four other parameters were allowed to vary with excitation power. While $\beta$ and phonon lifetimes are further discussed in the main text, we have included the data regarding $\alpha$ and $\tau_{\text {cool }}$ in Figure S1a and S1b, respectively.
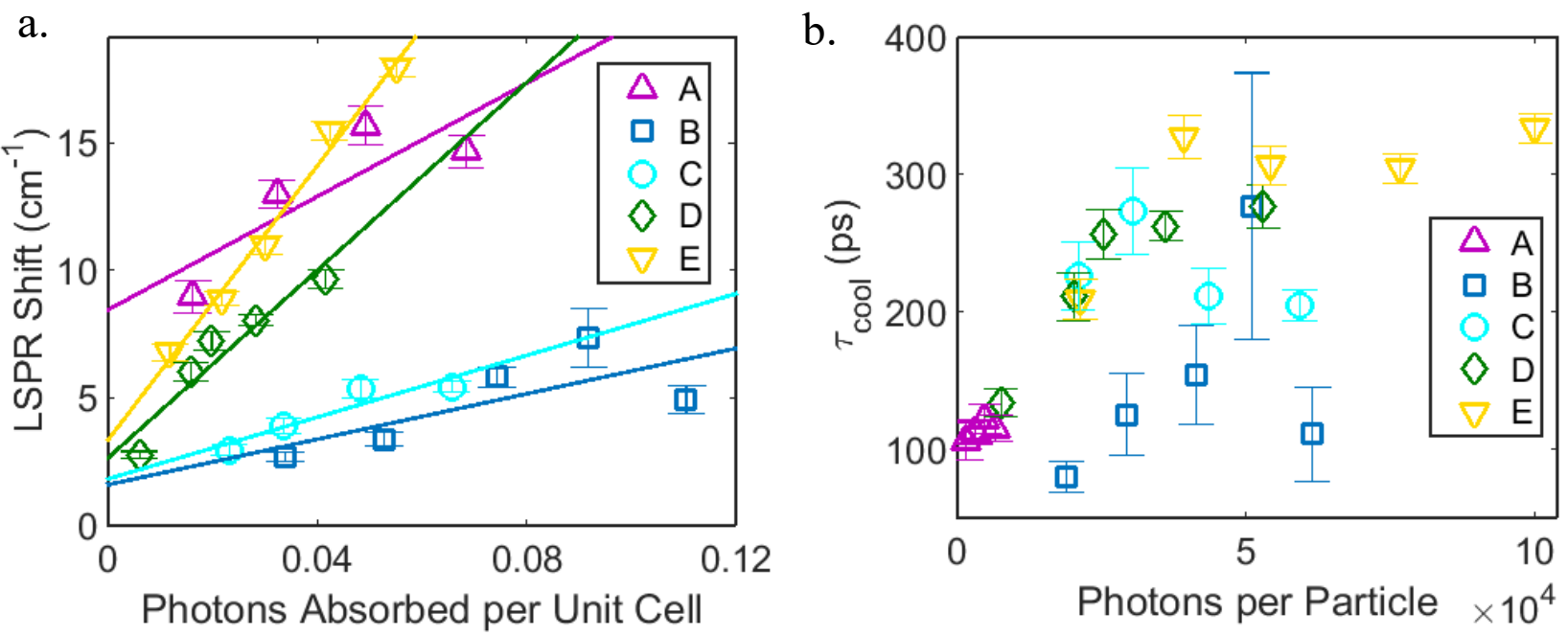

Error bars here and in the main text represent $95 \%$ confidence intervals from our nonlinear least squares fitting algorithm.

Figure S1. (a) LSPR shift from lattice heating. (b) Fitted lattice cooling lifetimes.

\section{Additional Damping Rate Information}

The observed phonon coherence dephasing comprises several components. Damping arises from sample inhomogeneity $\left(\tau_{2}\right)$ as particles of different sizes in the sample will exhibit different phonon frequencies that cause oscillations to eventually become out of phase leading to an apparent damping effect. This process is well approximated by a Gaussian decay profile provided that the particle size distribution is Gaussian. ${ }^{\mathrm{S} 1}$ This lifetime can be approximated as $\tau_{2} \approx T^{2} / \sqrt{ } 2 \pi \sigma_{T}$ where $\sigma_{T}$ is the standard deviation of the periods in the bipyramid sample with a mean period $T .^{\mathrm{S} 1}$ The approximation $T / \sigma_{T} \approx L / \sigma_{L}$, where $L$ and $\sigma_{L}$ are the mean and standard deviation in particle length, is often applied to directly utilize distributions from TEM measurements. ${ }^{\mathrm{S} 3, \mathrm{~S} 4} \mathrm{By}$ using this methodology, we were able to improve the accuracy of fitting $\tau_{l}$, the remaining so-called homogenous decay processes which are due to energy loss. To compare decay constants between materials with drastically different oscillation periods, we utilize a quality factor, $Q=\pi \tau_{1} / T$, which is the decay lifetime normalized by oscillation period. We used the model developed by Pelton et $\mathrm{al}^{\mathrm{S} 2, \mathrm{~S} 3}$ to separate $\tau_{1}$ into fluid damping (caused by solvent-phonon coupling) and intrinsic damping $\tau_{i}$ (caused by mechanisms within the nanoparticle). Briefly, based on particle size and geometry, the rate of damping caused by solvent interactions, $Q_{\text {fluid }}$, can be calculated. Once that coupling rate is determined, the intrinsic quality factor, $Q_{i n t}$, can be determined by the equation:

$$
1 / Q_{1}=1 / Q_{\text {fluid }}+1 / Q_{\text {int }}
$$


We consider this intrinsic quality factor as it removes effects caused by differences in solventphonon coupling and offers comprehension of processes that occur within the various nanoparticles.

In Figures S2 and S3 (which display the same data but different scale bars) we plot the dependence of $Q_{i n t}$ and $Q_{1}$ on pump intensity. Since the data comprises two decay terms (inhomogeneous and homogenous) it is possible for one of those decay terms to dominate the dynamics. Because the inhomogeneous damping term is determined by TEM images, it is constant throughout the fitting process. However, for several powers in samples A and C, the homogeneous lifetime was significantly higher than the inhomogeneous lifetime preventing effective parameter fitting. For these instances, only a lower error bars is plotted since this indicates the lower bound of that quality factor. However, the upper bound of this parameter or even its precise value cannot be accurately estimated. Therefore, while sample A might appear to exhibit relatively low quality factors, that is more a result of the sample inhomogeneity rather than actual low quality factors. To make this clearer, we include inhomogeneous lifetime normalized by oscillation period $\left(Q_{i n h}=\pi \tau_{2} / T\right)$. Additionally, $Q_{\text {fluid }}$ is included in these Figures to offer a sense of its relative effect.

Prior reports pointed to a linear relationship between excitation power and $Q_{1}{ }_{1}^{\mathrm{S} 2 \mathrm{~S} 3}$ However, we experimentally observe that quality factors plateau at higher excitation fluxes. It is unlikely that particle melting causes this trend as we undertook careful efforts to minimize degradation as discussed in Figure S5. The origin of this relationship is unclear to us, but may potentially be caused by heating changing the solvent viscosity. ${ }^{\mathrm{S} 2}$ It is worth noting that we observe higher quality factors in water than in previous work for a small bipyramid. ${ }^{\mathrm{S} 2 \mathrm{~S} 4}$ This trend could be explained by the fact that the prior effort calculated a quality factor via linear extrapolating to zero power. Since quality factors increase with excitation power, it follows that our reported values are larger.

Additionally, there seems to be a weak correlation between sample size and Qint with larger particles exhibiting smaller quality factors. The exception is sample A, which is explained above. Examining the TEMs we note that larger bipyramids exhibit rougher surfaces than smaller bipyramids, which could indicate lower crystalline quality leading to faster damping. This trend is demonstrated in the TEM images in Figures S4. The smaller bipyramids have boundaries that are clearly defined by straight lines and correspondingly have very high quality factors. However, the outline of the larger particles are distorted and as a result they have lower quality factors. If larger particles present lower crystallinity, rather than having larger size directly cause the lower quality factors this would explain why the trend is so weak. This dependence on crystallinity is consistent with what has been proposed regarding quality factors of nanorods; higher crystallinity corresponds to larger quality factors. ${ }^{\mathrm{S5}}$ These results suggest that it may be possible to increase phonon coherence lifetime by manipulating bipyramid crystallinity. It is worth noting, that we have also done additional analysis on less complete data sets which have yielded very similar results. 

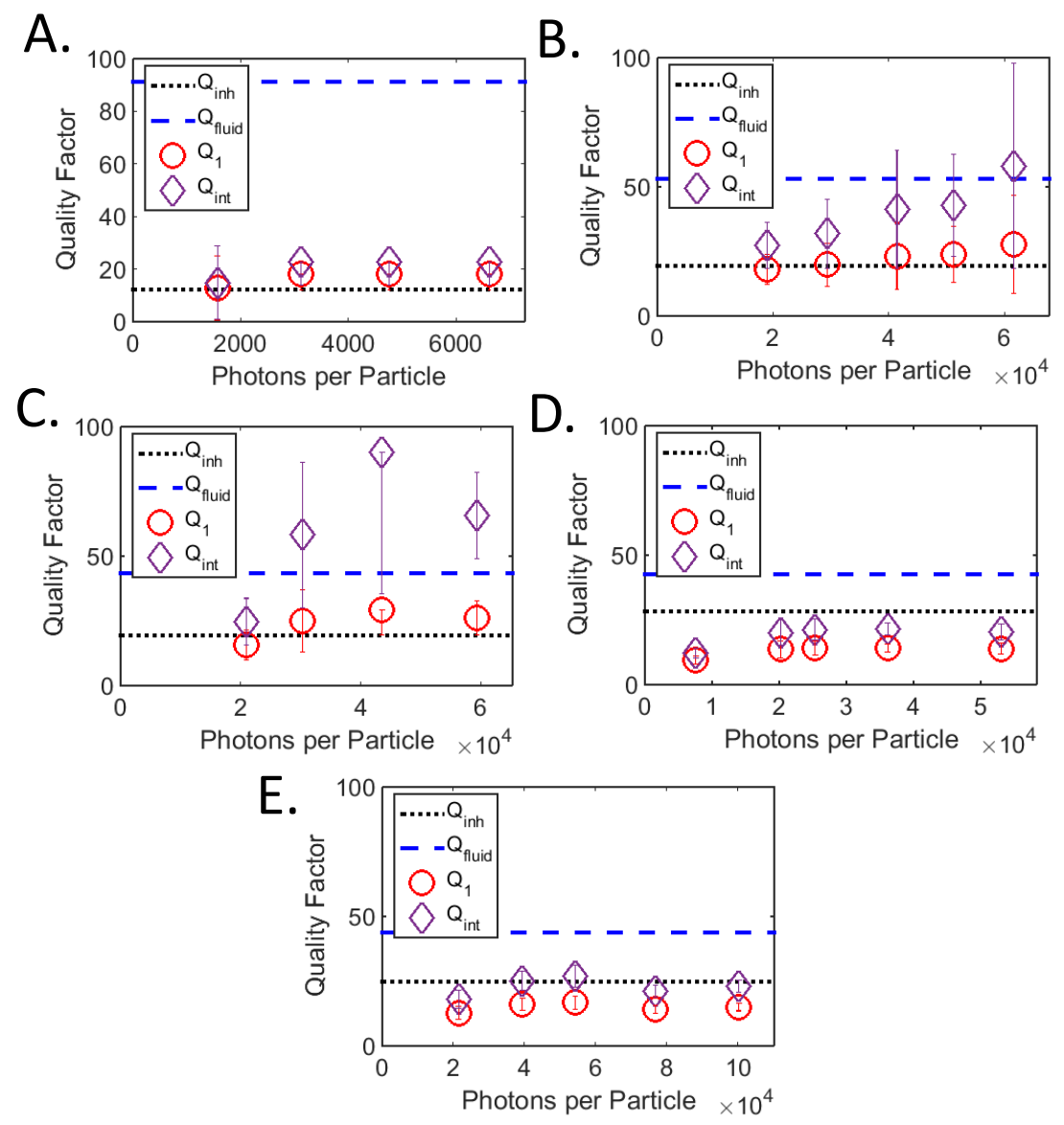

Figure S2. Various quality factors of samples A-E. 


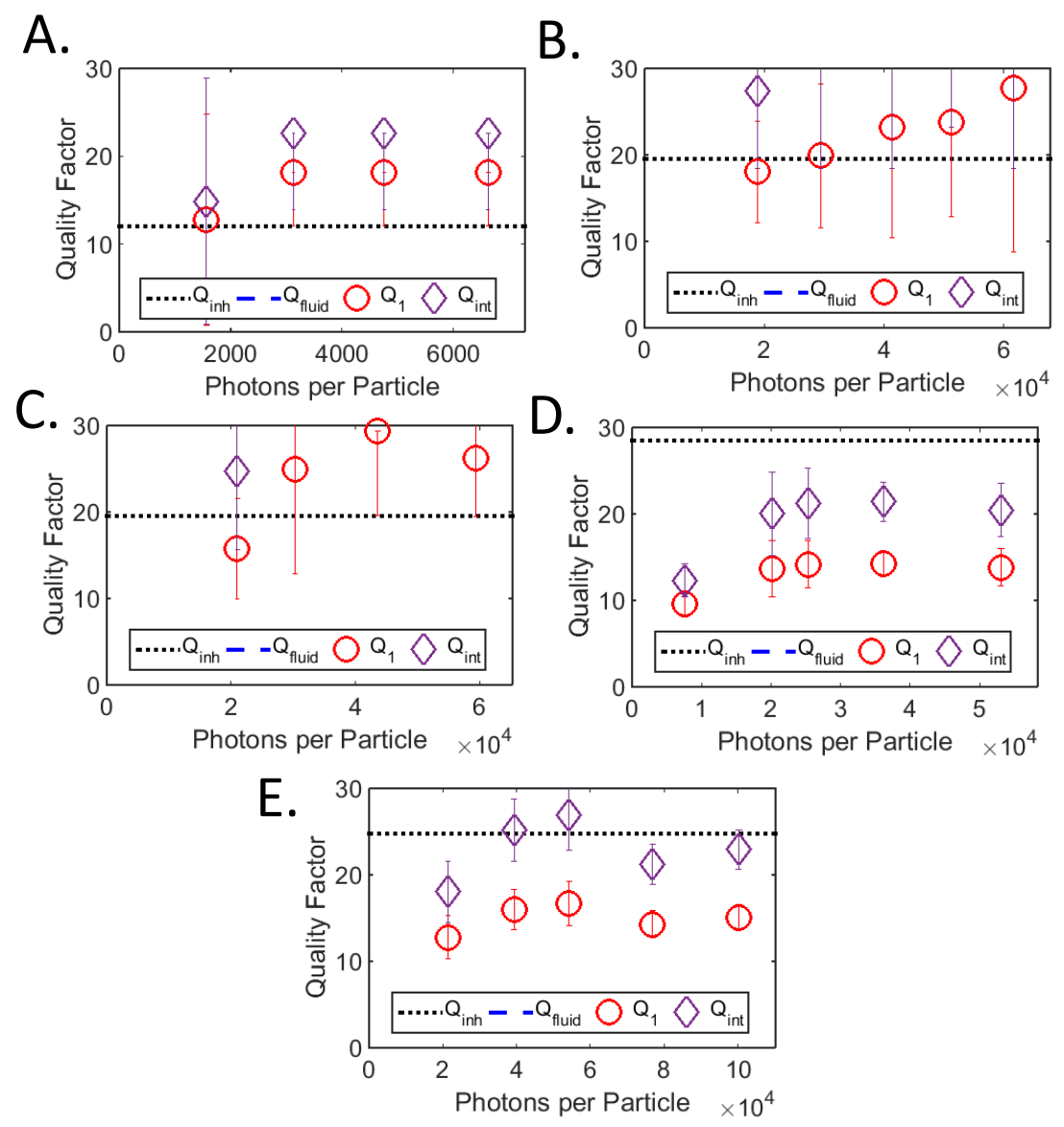

Figure S3. Zoomed-in sections of the data in Figure S2 

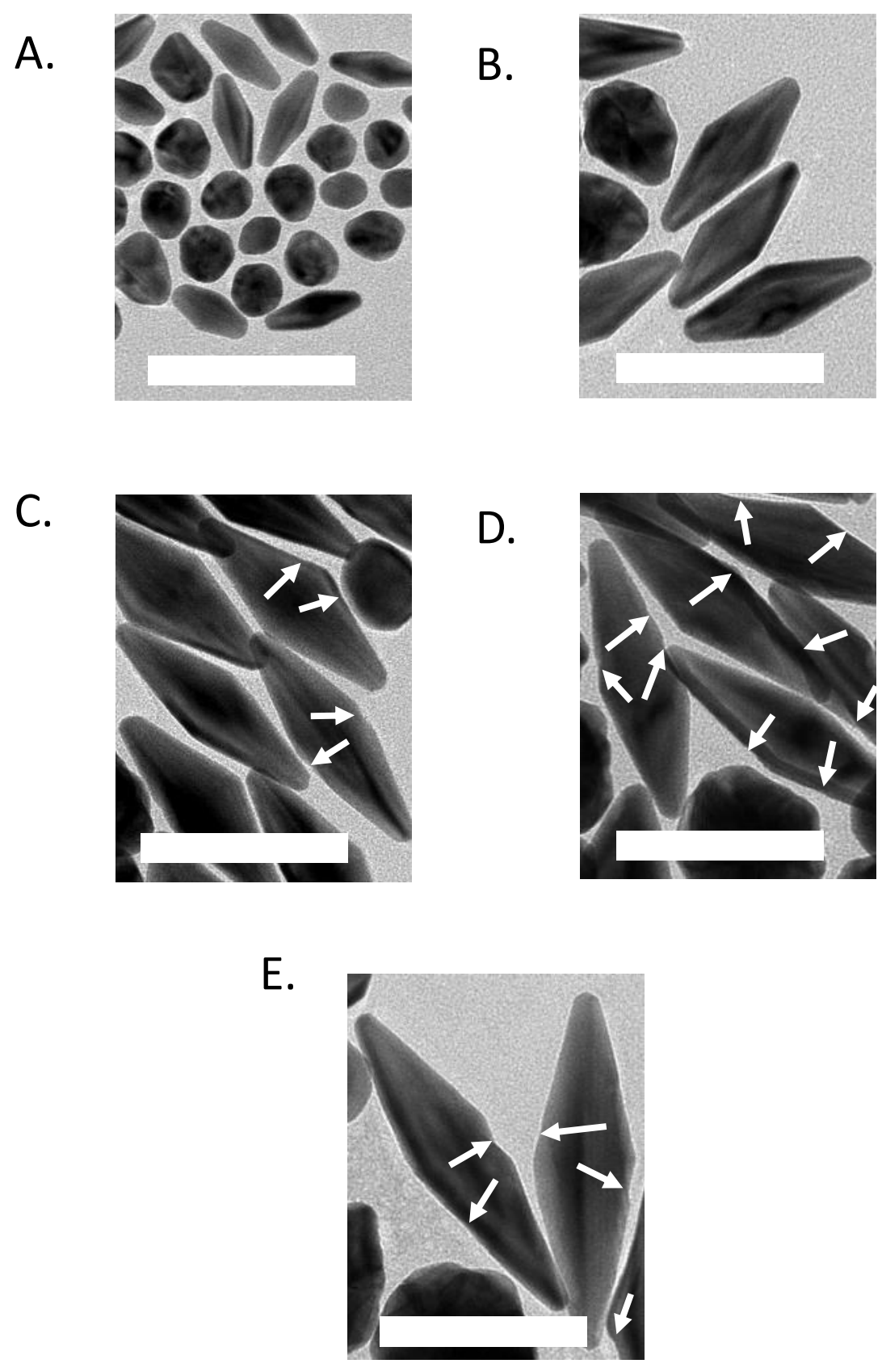

Figure S4. Larger view of TEM images of the bipyramid samples discussed in the main text. White scale bars represent $100 \mathrm{~nm}$. Arrows point to notable surface irregularities. 


\section{Sample Damage}

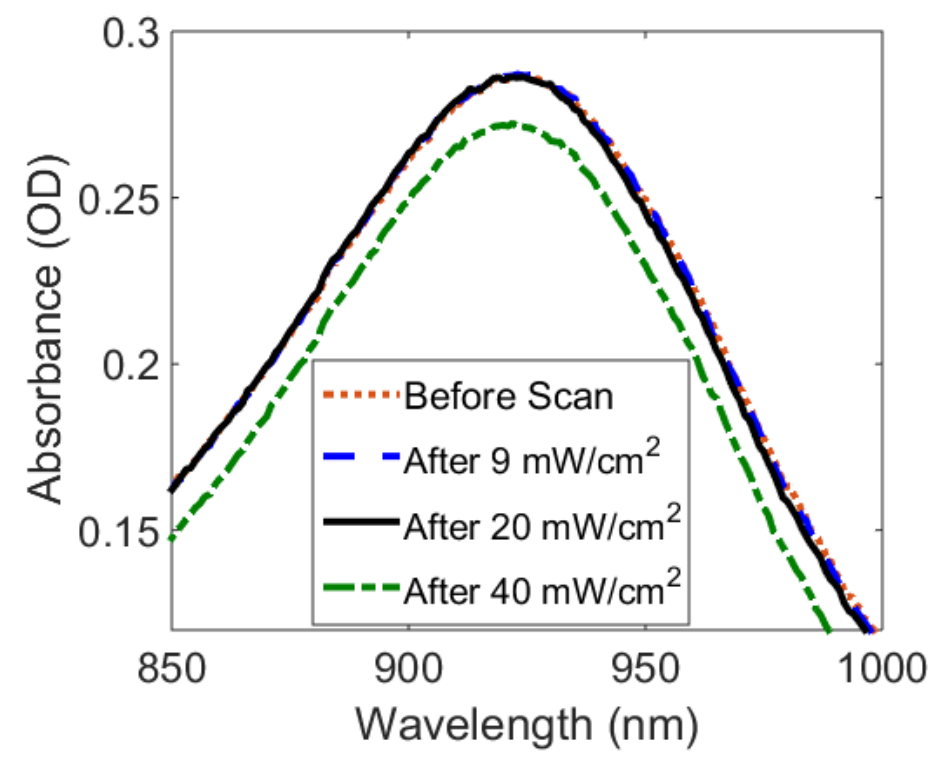

Figure S5. Extinction spectra of sample E before and after several transient absorption scans where $1 \mathrm{kHz}$ pump fluence is indicated. Probe fluence was negligible.

Solutions were rapidly stirred during transient optical experiments. To ensure we were measuring properties of the bipyramids rather than effects related to irreversible sample damage, we examined extinction spectra before and after each scan and only reproducible scans are examined. Figure S5 shows the spectra of sample E before and after several scans.

\section{Details of Sample Dimensions, Total Expansion, and Calculated Cross-Sections}

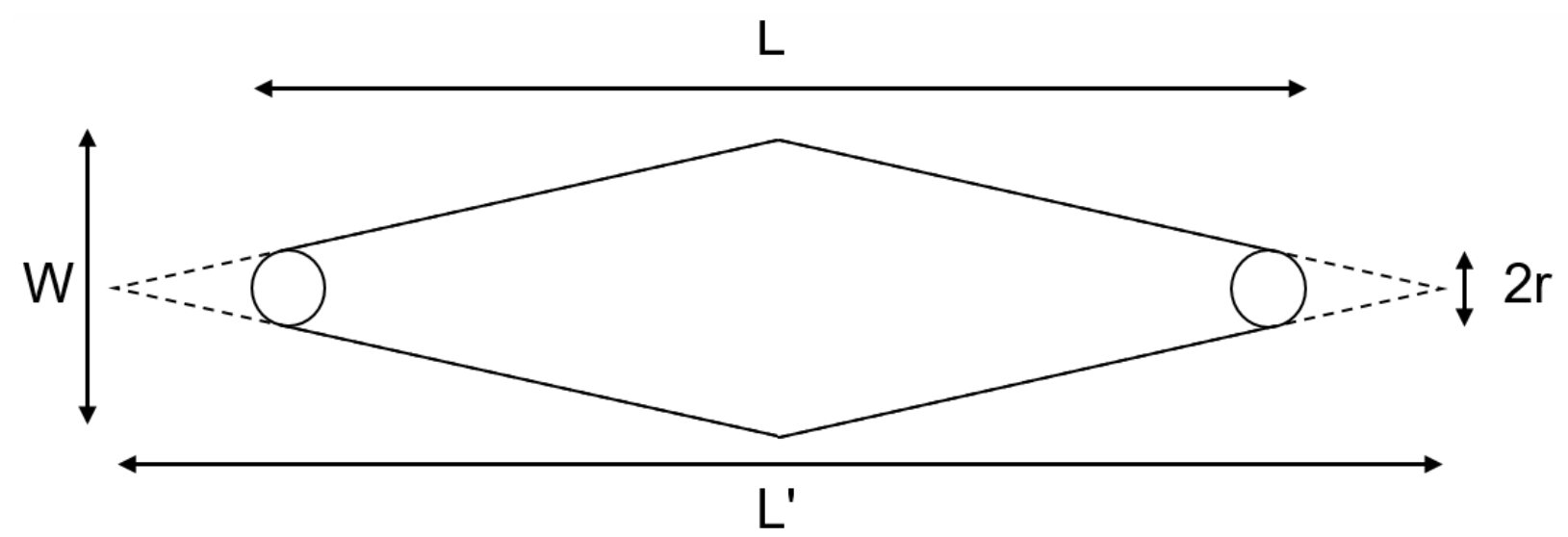

Figure S6. Bipyramid with relevant dimensions labeled. 
In addition to the particle length (L), width (W), and radius (r), an important parameter is projected length which is calculated geometrically for each particle using the formula:

$$
\begin{gathered}
\left(\mathrm{L}^{\prime}-(\mathrm{L}-2 \mathrm{r})\right) /(2 \mathrm{r})=\mathrm{L}^{\prime} / \mathrm{W} \\
\mathrm{L}^{\prime}\left(1-\frac{2 \mathrm{r}}{\mathrm{W}}\right)=\mathrm{L}-2 \mathrm{r} \\
\mathrm{L}^{\prime}=\frac{\mathrm{L}-2 \mathrm{r}}{\left(1-\frac{2 \mathrm{r}}{\mathrm{W}}\right)}
\end{gathered}
$$

The volume of the bipyramid is then calculated from the ensemble average using the formula:

$$
V=\frac{\pi L^{\prime}}{3}\left(\frac{W}{2}\right)^{2}+\frac{4 \pi r^{3}}{3}-2\left(\frac{\pi r^{2}}{3}\left(\frac{L^{\prime}}{2}-\frac{L}{2}+r\right)\right)
$$

From there, by using the density of gold, and the assumption of four atoms per unit cell, the number of unit cells is estimated.

Table S1. Bipyramid and nanojavelin sizes measured via TEM images.

\begin{tabular}{|c|c|c|c|c|c|}
\hline Sample & $\begin{array}{c}\text { Length } \\
(\mathrm{nm})\end{array}$ & $\begin{array}{c}\text { Width } \\
(\mathrm{nm})\end{array}$ & $\begin{array}{c}\text { Radius of } \\
\text { Curvature }(\mathrm{nm})\end{array}$ & $\begin{array}{c}\text { Aspect } \\
\text { Ratio }\end{array}$ & $\begin{array}{c}\text { Estimated Number } \\
\text { of Unit Cells }\end{array}$ \\
\hline A & $45.6 \pm 2.7$ & $18.7 \pm 0.9$ & $5.2 \pm 0.3$ & $2.45 \pm 0.14$ & $9.67 \times 10^{4}$ \\
\hline B & $94.0 \pm 3.4$ & $33.1 \pm 1.5$ & $6.8 \pm 0.4$ & $2.85 \pm 0.16$ & $5.57 \times 10^{5}$ \\
\hline C & $122.9 \pm 4.5$ & $38.3 \pm 2.9$ & $6.1 \pm 0.4$ & $3.23 \pm 0.28$ & $9.03 \times 10^{5}$ \\
\hline D & $146.4 \pm 3.6$ & $41.9 \pm 2.2$ & $6.3 \pm 0.5$ & $3.51 \pm 0.21$ & $1.28 \times 10^{6}$ \\
\hline E & $168.6 \pm 4.8$ & $46.6 \pm 3.5$ & $6.8 \pm 0.6$ & $3.64 \pm 0.29$ & $1.81 \times 10^{6}$ \\
\hline F & $139.9 \pm 5.9$ & $29.3 \pm 1.0$ & $7.5 \pm 0.6$ & $4.77 \pm 0.27$ & $7.70 \times 10^{5}$ \\
\hline G & $198.1 \pm 4.7$ & $33.0 \pm 1.3$ & $8.71 \pm 1.5$ & $6.01 \pm 0.30$ & $1.46 \times 10^{6}$ \\
\hline
\end{tabular}




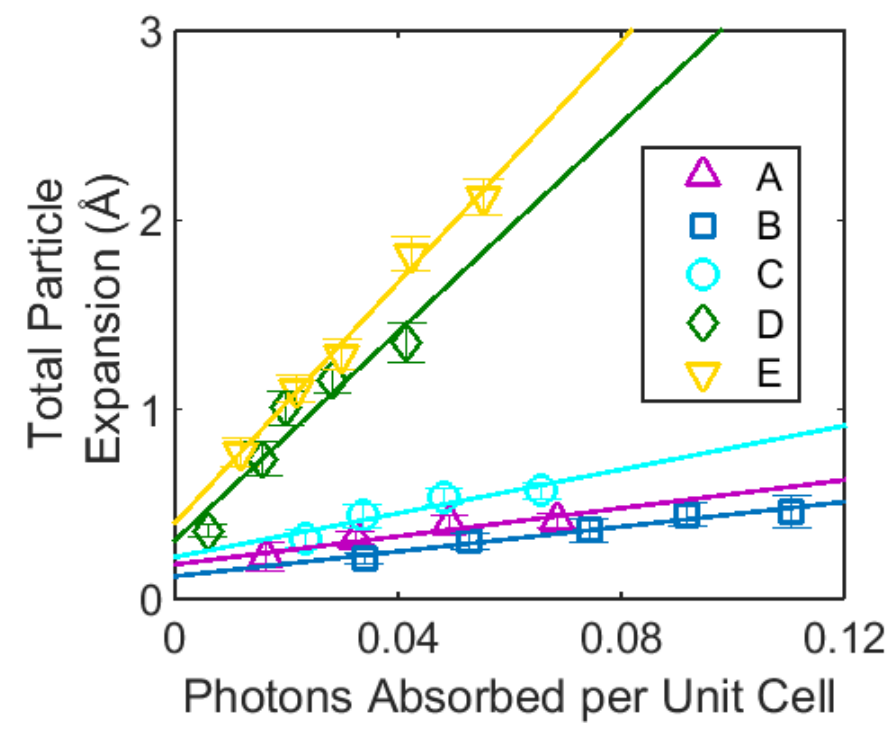

Figure S7. Total expansions of the particles from the lowest-order coherent acoustic phonon.

Table S2. Bipyramid Absorption and Scattering Cross Sections at $\lambda_{\max }$ from FDTD calculations

\begin{tabular}{|c|c|c|}
\hline Sample & Absorption Cross Section $\left(\mathrm{m}^{2}\right)$ & Scattering Cross Section $\left(\mathrm{m}^{2}\right)$ \\
\hline A & $7.74 \times 10^{-15}$ & $5.62 \times 10^{-16}$ \\
\hline B & $3.63 \times 10^{-14}$ & $2.01 \times 10^{-14}$ \\
\hline C & $4.74 \times 10^{-14}$ & $4.36 \times 10^{-14}$ \\
\hline D & $5.25 \times 10^{-14}$ & $6.00 \times 10^{-14}$ \\
\hline E & $5.46 \times 10^{-14}$ & $8.87 \times 10^{-14}$ \\
\hline
\end{tabular}

\section{References}

S1. Hartland, G. V. Coherent vibrational motion in metal particles: Determination of the vibrational amplitude and excitation mechanism. J. Chem. Phys. 2002 116, 8048-8055.

S2. Pelton, M.; Sader, J. E.; Burgin, J.; Liu, M.; Guyot-Sionnest, P.; Gosztola, D. Damping of acoustic vibrations in gold nanoparticles. Nat. Nano. 2009. 4, 492-495.

S3. Pelton, M.; Wang, Y.; Gosztola, D.; Sader, J. E. Mechanical Damping of Longitudinal Acoustic Oscillations of Metal Nanoparticles in Solution. J. Phys. Chem. C 2011 115, 23732-23740.

S4. Fernandes, B. D.; Spuch-Calvar, M.; Baida, H.; Tréguer-Delapierre, M.; Oberlé, J.; Langot, P.; Burgin, J. Acoustic Vibrations of Au Nano-Bipyramids and their Modification under Ag Deposition: a Perspective for the Development of Nanobalances. ACS Nano. 2013 7, 76307639.

S5. Ruijgrok, P. V., Zijlstra, P., Tchebotareva, A. L. \& Orrit, M. Damping of Acoustic Vibrations of Single Gold Nanoparticles Optically Trapped in Water. Nano. Lett. 2012 12, 1063-1069. 